\title{
Broadband Active Control of Transverse Scattering from All-Dielectric Nanoparticle
}

\author{
Huiwen $\mathrm{Yu}{ }^{1,2, \dagger}$, Hongjia Zhu ${ }^{1,2,+}$, Jinyang $\mathrm{Li}^{1,2}$, Zhaolong Cao ${ }^{1,2, *}$ and Huanjun Chen ${ }^{1,2}$ \\ 1 School of Electronics and Information Technology, Sun Yat-sen University, Guangzhou 510275, China; \\ yuhw3@mail2.sysu.edu.cn (H.Y.); zhuhj3@mail2.sysu.edu.cn (H.Z.); lijy293@mail2.sysu.edu.cn (J.L.); \\ chenhj8@mail.sysu.edu.cn (H.C.) \\ 2 State Key Lab of Optoelectronic Materials and Technologies, Guangdong Province Key Laboratory of Display \\ Material and Technology, Sun Yat-sen University, Guangzhou 510275, China \\ * Correspondence: caozhlong@mail.sysu.edu.cn \\ + These Authors Contributed Equally.
}

Citation: Yu, H.; Zhu, H.; Li, J.; Cao,

Z.; Chen, H. Broadband Active Control of Transverse Scattering from All-Dielectric Nanoparticle. Crystals 2021, 11, 920. https://doi.org/ $10.3390 /$ cryst 11080920

Academic Editor: Borislav Angelov

Received: 14 July 2021

Accepted: 4 August 2021

Published: 7 August 2021

Publisher's Note: MDPI stays neutral with regard to jurisdictional claims in published maps and institutional affiliations.

Copyright: (c) 2021 by the authors. Licensee MDPI, Basel, Switzerland. This article is an open access article distributed under the terms and conditions of the Creative Commons Attribution (CC BY) license (https:// creativecommons.org/licenses/by/ $4.0 /)$

\begin{abstract}
Steering electromagnetic scattering by subwavelength objects is usually accompanied by the excitation of electric and magnetic modes. The Kerker effect, which relies on the precise overlapping between electric and magnetic multipoles, is a potential approach to address this challenge. However, fundamental limitations on the reconfigurability and tunability challenge their future implementation in practical applications. Here, we demonstrate a design approach by applying coherent control to a silicon nanodisk. By utilizing an experimentally feasible two-wave excitation, this coherent light-by-light control enables a highly reconfigurable, broadband, and tunable transverse scattering, extending the feasibility of unidirectional scattering in various practical scenarios, including on-chip integrations and optical communications.
\end{abstract}

Keywords: Mie resonator; coherent control; electric dipole resonance; magnetic dipole resonance; Kerker effect; unidirectional scattering; transverse scattering; interference

\section{Introduction}

Precise control over the scattering property of subwavelength nanostructure lies at the heart of nanotechnology [1,2]. For this purpose, the Kerker effect, which exploits the far-field interference between electric and magnetic multipoles, is widely adopted for unidirectional scattering [3-5]. Multipolar nanophotonics is closely associated with highindex dielectric materials owning to their suppressed dissipation losses and compatibility with modern CMOS techniques [6-8]. By judiciously engineering the geometry of dielectric nanoparticles, light can be unidirectionally scattered to forward [4,9], backward [4], or even transverse $[10,11]$ directions. Currently, the study on unidirectional scattering mainly focuses on the design of natural resonant modes (or eigenstates) inside a nanostructure, which depends heavily on the geometry and the working wavelengths. For example, the electric dipole (ED) and magnetic dipole (MD) responses from a silicon nanosphere are dispersive, thereby resulting in an extremely narrow spectral window for it to act as a Huygens' source [12]. Moreover, it is reported that the precise overlapping between higherorder electric and magnetic multipolar resonances leads to side-directed scattering, but at the cost of ultranarrow bandwidth, delicate nanostructure design, and stringent fabrication requirements [11]. In addition, the lack of reconfigurability for given nanostructure also hinders substantial developments for nanodevices with miscellaneous optical functions.

In contrast to the eigenstate-oriented design approach, the recently emerged concept of 'coherent control' suggests an on-demand strategy for reconfigurable unidirectional scattering [13]. In the context of nanophotonics, coherent light-by-light control refers to the excitation of nanostructures in interference fields, which can be constructed by, for example, multiple coherent plane waves [14,15], radially or azimuthally polarized lights, 
etc. [16,17]. By tuning the intensity and phase difference between incident lights, one can continuously tune the optical response of a static nanostructure noninvasively $[14,15,18,19]$, enabling a variety of applications in ultrafast and nonlinear optics such as displacement sensing [20,21], optical force [22], coherent perfect absorption [23], and on-chip optoelectronic applications [24,25], etc.

In this letter, we apply coherent control to a silicon nanodisk and demonstrate a highly reconfigurable, broadband, and tunable transverse scattering without utilizing higher-order multipoles. Specifically, by adjusting the polarization states and phase difference between two coherent plane waves, the out-of-plane MD $\left(m_{\perp}\right)$ and in-plane ED $\left(p_{\|}\right)$moments from the silicon nanodisk are independently excited. Such coherent excitation enables the continuous tuning between ED and MD modes, thereby extending unidirectional scattering to all transverse angles and broadband spectra regions. Our results provide a simple way for reconfigurable scattering manipulation without moving the excitation beams, which may find applications in on-chip integrations and optical communications.

\section{Principle of Coherent Control for Transverse Scattering}

We start by discussing the coherent excitation of individual dipole mode. In general, the Kerker effect takes place in the presence of ED and MD modes, where their dipole moments should be (i) orthogonally oriented, (ii) in-phase or out-of-phase, and (iii) have equal magnitude. For conventional studies where a nanosphere is impinged by a plane wave, the first criterion is inherently fulfilled. However, as the ED and MD modes are simultaneously excited by a single beam, their dipole moments are determined by the electric and magnetic dipolar polarizabilities, which cannot always be precisely matched to fulfilled the latter two conditions. To overcome this fundamental limitation, coherent radially and azimuthally polarized beams with orthogonal incidence are investigated [16]. In this case, the ED mode of a nanosphere can only be excited by focused radially polarized light, while its MD counterpart can only be excited by focused azimuthally polarized light [16]. However, as the construction of radially/azimuthally polarized beams relies on two bulky high numerical aperture objectives, this approach is less practical from an experimental point of view. Herein, we propose a different approach to address this issue, which involves two plane waves impinging on a Mie resonator (Figure 1). We should emphasize that this configuration is experimentally feasible, for example, by incorporating Fourier space microscopy and interferometer setup (see Figure S1 in the Supplementary Material for a schematic diagram of the excitation setup) $[17,26]$.

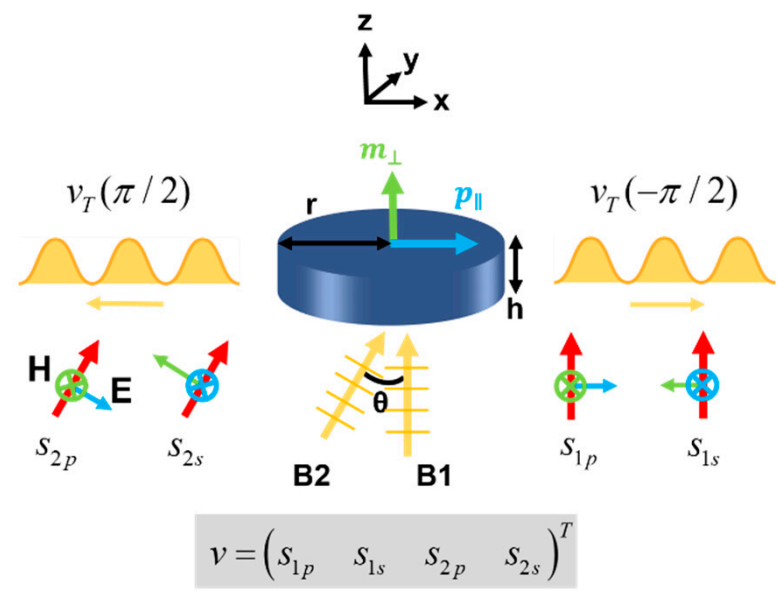

Figure 1. Schematic of the coherent control approach. A nanodisk with radius $r$ and height $h$ is located at the origin of the Cartesian coordinate system. Two coherent beams illuminate normally (B1) and obliquely (B2) towards the nanodisk. The incident angle of the oblique beam is $\theta$. The excitation state of the incident beams, according to their polarizations, can be expressed as a vector 
$v=\left(\begin{array}{llll}s_{1 p} & s_{1 s} & s_{2 p} & s_{2 s}\end{array}\right)^{T}$. The red, blue, and green arrows indicate the propagation direction, electric field, and magnetic field of the plane waves, respectively. The Kerker condition, once fulfilled by coherent excitation, enables all-angle transverse scattering.

Based on this approach, we consider a silicon nanodisk located at the origin of the Cartesian coordinate system. When the size of the nanodisk is small compared to the wavelength, its ED and MD response can be expressed in terms of anisotropic polarizabilities $\boldsymbol{p}=P_{e} \boldsymbol{E}(0)$ and $\boldsymbol{m}=P_{m} \boldsymbol{H}(0)$, where $P_{e}$ and $P_{m}$ are electric and magnetic polarizability tensors, and $\boldsymbol{E}(0)$ and $\boldsymbol{H}(0)$ are the electric and magnetic fields at origin, respectively. The two coherent plane waves illuminate normally (denote as B1) and obliquely (denote as B2) towards the nanodisk, which is defined as an excitation vector $v=\left(\begin{array}{cccc}s_{1 p} & s_{1 s} & s_{2 p} & s_{2 s}\end{array}\right)^{T}$. The incident angle of B2 wave is $\theta$. Symbols $s_{1 p}, s_{1 s}, s_{2 p}$, and $s_{2 s}$ are the p-and s-polarized components of each beam, respectively. Therefore, the interplay between coherent sources leads to a flexible design of the interference field at origin, which can be expressed as,

$$
\begin{gathered}
\boldsymbol{E}(0)=E_{0}\left(\begin{array}{cccc}
1 & 0 & \cos \theta & 0 \\
0 & 1 & 0 & 1 \\
0 & 0 & -\sin \theta & 0
\end{array}\right) v, \\
\boldsymbol{H}(0)=\frac{E_{0}}{\eta}\left(\begin{array}{cccc}
0 & -1 & 0 & -\cos \theta \\
1 & 0 & 1 & 0 \\
0 & 0 & 0 & \sin \theta
\end{array}\right) v,
\end{gathered}
$$

where $E_{0}$ and $\eta$ are the incident electric field and the impedance of the environment, respectively. One can notice from Equations (1) and (2) that there exist two particular interference fields for independent excitation. First, $v_{E}(\alpha)=\left(\begin{array}{cccc}\cos \alpha & \sin \alpha & 0 & 0\end{array}\right)^{T}$ corresponds to in-plane electric field $\left(E(0)=E_{0}\left(\begin{array}{ccc}\cos \alpha & \sin \alpha & 0\end{array}\right)^{T}\right)$ along $\alpha$ direction and in-plane magnetic field $\left(\boldsymbol{H}(0)=E_{0} / \eta\left(\begin{array}{lll}-\sin \alpha & \cos \alpha & 0\end{array}\right)^{T}\right)$ along $\alpha+\pi / 2$ direction, where $\alpha$ is the incident polarization angle with respect to the $x$-axis. Second, $v_{H}=1 / \sqrt{2}\left(\begin{array}{cccc}0 & 1 & 0 & -1\end{array}\right)^{T}$ corresponds to a pure magnetic field $(\boldsymbol{E}(0)=0$ and $\left.H(0)=E_{0} / \eta\left(\begin{array}{ccc}\cos \theta-1 & 0 & -\sin \theta\end{array}\right)^{T}\right)$ at the origin. In general, all the nonzero field components from $v_{E}(\alpha)$ and $v_{H}$ are possible to excite the dipolar modes. However, if a nanodisk is rationally designed to dominantly support $p_{\|}$and $m_{\perp}$ modes (that is, $P_{e}=\operatorname{diag}\left(\begin{array}{ccc}\alpha_{e} & \alpha_{e} & 0\end{array}\right)$ and $P_{m}=\operatorname{diag}\left(\begin{array}{ccc}0 & 0 & \alpha_{m}\end{array}\right)$, where $\alpha_{e}$ and $\alpha_{m}$ are frequencydependent electric and magnetic polarizabilities, respectively), the $v_{E}(\alpha)$ and $v_{H}$ excitations are decoupled. This can be manifested by incorporating $P_{e}$ and $P_{m}$ with Equations (1) and (2), which writes,

$$
\begin{gathered}
\boldsymbol{p}(\alpha)=P_{e} \boldsymbol{E}(0)=E_{0}\left(\begin{array}{cccc}
\alpha_{e} & 0 & \alpha_{e} \cos \theta & 0 \\
0 & \alpha_{e} & 0 & \alpha_{e} \\
0 & 0 & 0 & 0
\end{array}\right) v, \\
\boldsymbol{m}=P_{m} \boldsymbol{H}(0)=\frac{E_{0}}{\eta}\left(\begin{array}{cccc}
0 & 0 & 0 & 0 \\
0 & 0 & 0 & 0 \\
0 & 0 & 0 & \alpha_{m} \sin \theta
\end{array}\right) v,
\end{gathered}
$$

Equations (3) and (4) can be reorganized by introducing the reduced excitation matrix $P$ given by,

$$
\left(\begin{array}{c}
p_{x} \\
p_{y} \\
m_{z}
\end{array}\right)=P v=E_{0}\left(\begin{array}{cccc}
\alpha_{e} & 0 & \cos \theta \alpha_{e} & 0 \\
0 & \alpha_{e} & 0 & \alpha_{e} \\
0 & 0 & 0 & \sin \theta \alpha_{m} / \eta
\end{array}\right) v
$$


where $p_{x}, p_{y}$, and $m_{z}$ are the ED moments along the $\mathrm{x}$ - and $\mathrm{y}$-axis, and MD moment along the z-axis, respectively. By applying $v_{E}(\alpha)$ and $v_{H}$ to Equation (5), one arrives at the individual excitation of each mode,

$$
\begin{gathered}
\left(\begin{array}{c}
p_{x} \\
p_{y} \\
m_{z}
\end{array}\right)=P v_{E}(\alpha)=E_{0} \alpha_{e}\left(\begin{array}{c}
\cos \alpha \\
\sin \alpha \\
0
\end{array}\right), \\
\left(\begin{array}{c}
p_{x} \\
p_{y} \\
m_{z}
\end{array}\right)=P v_{H}=-\frac{\sin \theta \alpha_{m}}{\sqrt{2}} \frac{E_{0}}{\eta}\left(\begin{array}{l}
0 \\
0 \\
1
\end{array}\right) .
\end{gathered}
$$

Therefore, $v_{E}(\alpha)$ exclusively guides the orientation of in-plane ED resonance along the $\alpha$ direction, whereas $v_{H}$ can only excite the out-of-plane magnetic dipole mode. Based on the individual excitation condition, the Kerker condition, therefore, can be fulfilled by matching the ED and MD moments, which writes,

$$
\left(\begin{array}{c}
p_{x} \\
p_{y} \\
\sqrt{\varepsilon \mu} m_{z}
\end{array}\right)=P\left(\zeta_{e} v_{E}(\alpha)+\sqrt{\varepsilon \mu} \zeta_{m} v_{H}\right) \propto\left(\begin{array}{c}
\cos \alpha \\
\sin \alpha \\
1
\end{array}\right)
$$

where $\varepsilon, \mu, \zeta_{e}$, and $\zeta_{m}$ are the permittivity and permeability of the environment, amplitude for $v_{E}(\alpha)$ and $v_{H}$ excitation, respectively. Solving Equation (8) one arrives at the transverse scattering condition for the nanodisk,

$$
\frac{\zeta_{e}}{\zeta_{m}}=-\frac{\sin \theta}{\sqrt{2}} \frac{\alpha_{m} \varepsilon}{\alpha_{e}} .
$$

Therefore, by denoting the amplitude ratio $\kappa=\zeta_{e} / \zeta_{m}$, the transverse scattering condition is fulfilled under the excitation,

$$
v_{T}(\alpha)=\kappa v_{E}(\alpha)+v_{H}
$$

Equation (10) is the central result of this work. We should emphasize that, since the scattering direction is perpendicular to both $p_{\|}$and $m_{\perp}$ modes, the parameter $\alpha$ provides a full $2 \pi$ transverse scattering coverage. That is, given a specific polarization angle $\alpha$, light will be exclusively scattered to the $\alpha+\pi / 2$ direction. In addition, the applicability of Equation (10) can in principle cover a broadband spectral range, as long as the sole prerequisite that $p_{\|}$and $m_{\perp}$ modes are the leading resonances is still valid.

\section{Results and Discussion}

We carry out finite-difference time-domain (FDTD) method (Lumerical Inc., Vancouver, BC, Canada) to validate the theoretical predictions. We first design the high-index resonator with in-plane ED and out-of-plane MD modes. In general, the MD resonance results from the circular displacement currents inside the particle, while the ED resonance is due to the oscillating bound charges [27]. Therefore, it is convenient to tune the resonance overlap between ED and MD modes simply by adjusting the disk height-toradius aspect ratio [28]. Figure 2 a plots the scattering cross section $\sigma_{\text {scat }}$ of a single Si nanodisk suspended in the air. The disk height $h$ is ranging from 10 to $90 \mathrm{~nm}$ while the radius $r$ is fixed to $110 \mathrm{~nm}$. The nanodisk is illuminated by B2 with the incident angle defined by $\theta=\arcsin \left(\lambda \sin \theta_{0} / \lambda_{0}\right)$, where $\lambda_{0}=533.7 \mathrm{~nm}$ and $\theta_{0}=30^{\circ}$ (we denote as $v_{0}=\left(\begin{array}{llll}0 & 0 & 0 & 1\end{array}\right)^{T}$ excitation). To better analyze the ED and MD modes, we use the freeware MENP to perform exact multipolar decompositions of the disk responses [29,30]. Their traced spectral maxima position of the dipole moments are superimposed as white dashed lines. The two resonance modes, labeled as $p_{y}$ and $m_{z}$, show a clear increase in $\sigma_{\text {scat }}$ spectra for increasing $h$. The increase in $\sigma_{\text {scat }}$ spectra can be attributed to the increase 
of particle volume and hence their polarizability. On the other hand, the resonance positions of the ED and MD modes experience a strong red-shift by increasing $h$, which is in sharp contrast to plasmonic nanoparticles where the increase in $h$ gives rise to the blue shift for in-plane dipole mode. The observations in Figure $2 \mathrm{a}$ are consistent with the literature [31,32], which can be analytically estimated by the magnetic wall boundary condition [33]. In addition, the resonance peaks positioned at $h>50 \mathrm{~nm}$ and $\lambda<550 \mathrm{~nm}$ are due to the excitation of higher-order ED, MD, and magnetic quadrupole modes. For the purpose of this study, we restrict our coherent control hereafter to the $h<50 \mathrm{~nm}$ disks. The black line in Figure $2 \mathrm{~b}$ plots the crosscut of the vertical dotted line in Figure 2a, which corresponds to the $\sigma_{\text {scat }}$ spectrum of a Si disk with $h=40 \mathrm{~nm}$ and $r=110 \mathrm{~nm}$. As expected, two clear peaks, which correspond to the ED mode at $\lambda=552 \mathrm{~nm}$ and the MD mode at $\lambda=632 \mathrm{~nm}$, are simultaneously excited. The electric and magnetic polarizabilities for the Si disk, defined as the ratio of the multipolar moments to the electromagnetic fields at origin, are plotted in Figure $2 \mathrm{c}$ for comparison (i.e., $\alpha_{e, \|}=p_{x} / E_{x}(0), \alpha_{e, \perp}=p_{z} / E_{z}(0)$ from $v=\left(\begin{array}{cccc}0 & 0 & 1 & 0\end{array}\right)^{T}$ excitation and $\alpha_{m, \|}=m_{x} / H_{x}(0), \alpha_{m, \perp}=m_{z} / H_{z}(0)$ from $v=\left(\begin{array}{llll}0 & 0 & 0 & 1\end{array}\right)^{T}$ excitation, where $\alpha_{e, \|}$ and $\alpha_{e, \perp}\left(\alpha_{m, \|}\right.$ and $\left.\alpha_{m, \perp}\right)$ are the in-plane and out-of-plane electric (magnetic) polarizabilities, respectively). As one can see, $\alpha_{e, \|}$ and $\alpha_{m, \perp}$ are indeed the dominant polarizabilities, ensuring the applicability of the coherent control equations. We then conduct coherent control to excite these two modes independently. The coherent excitation in FDTD can be implemented by simultaneously introducing two total-field scattered-field sources during simulation. The $\sigma_{\text {scat }}$ spectra of the Si nanodisk under $v_{E}(\pi / 2)$ and $v_{H}$ excitations are depicted as blue and green lines in Figure $2 \mathrm{~b}$. The far-field $\sigma_{\text {scat }}$ spectra clearly reveal that, $v_{E}(\pi / 2)$ exclusively excites the ED mode, while $v_{H}$ excitation yields a single MD resonance peak. The individual excitation can in addition be confirmed by investigating their far-field radiation patterns. Figure $2 \mathrm{~d}-\mathrm{f}$ plot the nanodisk radiation patterns in the $\mathrm{XY}$ plane for $v_{0}, v_{E}(\pi / 2)$ and $v_{H}$ excitations. The radiation wavelength is chosen to $633 \mathrm{~nm}$ (HeNe laser) for experimental consideration, which corresponds to the black, blue, and green arrows in Figure $2 b$, respectively. As one can see, when the nanodisk is illuminated by the $v_{0}$ incidence (black arrow, Figure $2 \mathrm{~b}$ ), both $p_{y}$ and $m_{z}$ dipoles are excited, leading to the far-field interference and therefore a small tail in the $-x$ direction (Figure $2 \mathrm{~d}$ ). In contrast, $v_{E}(\pi / 2)$ and $v_{H}$ give rise to the exclusive excitations for fundamental y-polarized ED and z-polarized MD modes, unambiguously confirming the coherent excitation principle we developed.

(a)

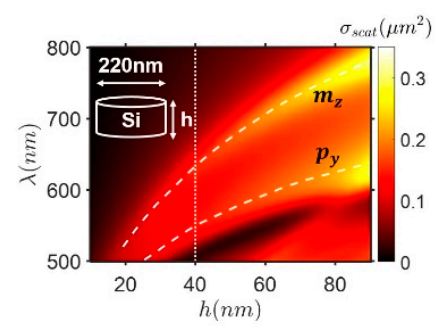

(d)

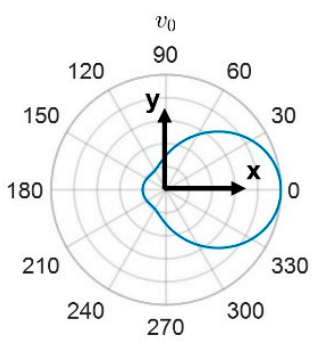

(b)

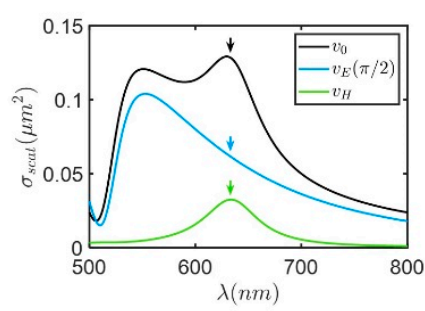

(e)

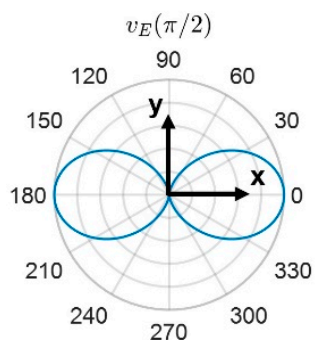

(c)

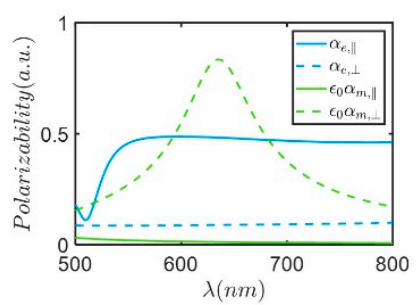

(f)

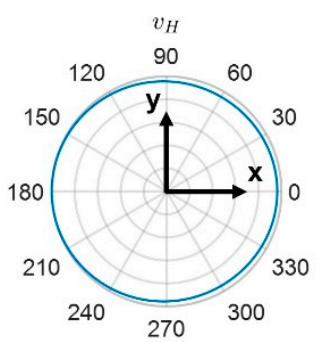

Figure 2. The optical response of a nanodisk under single beam and coherent excitation. (a) $\sigma_{\text {scat }}$ 
spectra of a single nanodisk with height ranging from 10 to $90 \mathrm{~nm}$. The disk radius is fixed to $110 \mathrm{~nm}$. The nanodisk is suspended in the air and is illuminated by $v_{0}$. The white dashed lines are the traced spectral maxima position of the dipole moment. (b) $\sigma_{\text {scat }}$ spectra of the $r=110 \mathrm{~nm}$ and $h=40 \mathrm{~nm}$ nanodisk under $v_{0}$ (black), $v_{E}(\pi / 2)$ (blue) and $v_{H}$ (green) excitations, respectively. (c) The electric and magnetic polarizabilities indicate that $\alpha_{e, \|}$ and $\alpha_{m, \perp}$ are indeed the dominant polarizability components. (d-f) The corresponding far-field radiation pattern for $v_{0}(\mathbf{d}), v_{E}(\pi / 2)(\mathbf{e})$ and $v_{H}(\mathbf{f})$ excitations.

With the individual excitation of ED and MD modes ready, we proceed to perform coherent control for unidirectional scattering. Two wavelengths, $600 \mathrm{~nm}$ and $633 \mathrm{~nm}$, are chosen here. Their vector amplitude ratio $\kappa$ is calculated to be $0.44 e^{-1.65 i}$ (at $600 \mathrm{~nm}$ ) and $0.72 e^{-2.27 i}$ (at $633 \mathrm{~nm}$ ) based on the electric and magnetic polarizabilities (Equation (9) and Figure 2c). To demonstrate the capability of Equation (10), we numerically calculate the far-field radiation pattern under $v_{T}(\alpha)$ excitation by considering three polarization angles: $\alpha=0, \pi / 4$, and $\pi / 2$, respectively. Their corresponding scattering directions, according to the Kerker condition, are expected along $-\pi / 2,-\pi / 4$, and 0 directions with respect to $\mathrm{x}$-axis, respectively. The simulated radiation patterns are shown in Figure 3, with Figure 3a corresponding to the operating wavelength at $600 \mathrm{~nm}$ and Figure $3 \mathrm{~b}$ corresponding to the operating wavelength at $633 \mathrm{~nm}$, respectively. The red, blue, and green arrows indicate the scattering direction, the orientation of ED and MD modes, respectively. As one can see, all coherent excitations exhibit complete suppression in the unwanted $(-\boldsymbol{k})$ direction. Even at the off-resonance region $(\lambda=600 \mathrm{~nm})$, Equation (10) faithfully provides the continuous routing to the full $2 \pi$ transverse scattering range with high efficiency. We have also calculated the directivity of the radiation using the definition:

$$
D=\frac{4 \pi}{\int_{0}^{2 \pi} \int_{0}^{\pi} F^{2}(\theta, \varphi) \sin \theta d \theta d \varphi}
$$

where $F(\theta, \varphi)$ is the radiated power in the direction $(\theta, \varphi)$, and $\theta, \varphi$ are the elevation and azimuthal angles, respectively. The corresponding directivities for Figure 3 are: 3.02 $(\alpha=0), 2.86(\alpha=\pi / 4)$, and $2.66(\alpha=\pi / 2)$ for $600 \mathrm{~nm}$ excitation, and $2.83(\alpha=0), 2.83$ $(\alpha=\pi / 4)$, and $2.85(\alpha=\pi / 2)$ for $633 \mathrm{~nm}$ excitation, respectively. Both of them are close to the theoretical directivity value $(D=3)$ of the Kerker condition. We should emphasize that, this approach can also be applied to higher multipolar modes for better directivity [2]. In addition, as the capability of the coherent control solely depends on the "dominance" of the $p_{\|}$and $m_{\perp}$ modes, it can be evaluated by the $\alpha_{e, \|} / \alpha_{e, \perp}$ and $\alpha_{m, \perp} / \alpha_{m, \|}$ information. The polarizability ratios are depicted in Figure S2a in the Supplementary Material, where their reasonably high values (exceed 3) are observed for wavelength larger than $520 \mathrm{~nm}$. We have simulated the radiation pattern for $v_{T}(0)$ excitation at wavelength $\lambda=510,520$, 530,730 , and $790 \mathrm{~nm}$. One clearly sees that for $\lambda=510 \mathrm{~nm}\left(\alpha_{e, \|} / \alpha_{e, \perp}=1.28\right)$, the radiation pattern shows a small tail along $\alpha=60^{\circ}$ direction (Figure S2b). However, as $p_{\|}$and $m_{\perp}$ modes prevail when $\lambda>520 \mathrm{~nm}$, the radiation patterns in Figure S2c-f exhibit efficiency transverse scatting, clearly confirming the applicability of coherent control methods.

Finally, we perform the coherent control in a realistic system by placing the Si nanodisk on the N-BK7 glass substrate $(\mathrm{n}=1.515)$. The nanodisk has the same geometry as in Figure 3. The system is further immersed in an index-matching oil to produce an optically homogeneous medium for the nanodisk. Following the same procedure described above, the vector amplitude ratio $\kappa$ is calculated to be $0.53 e^{-2.64 i}$ at $633 \mathrm{~nm}$. The numerically calculated radiation patterns with polarization angles $\alpha=0, \pi / 4$, and $\pi / 2$ are depicted in Figure 4. As expected, almost identical far-field profiles are observed, demonstrating the feasibility of continuous routing on realistic systems. In addition, since the continuous routing is based on the polarization angle $\alpha$, which can be readily implemented by, for example, rotational polarizers or spatial light modulators, the above tuning approach does not require any movement components in excitation beams. 
(a)

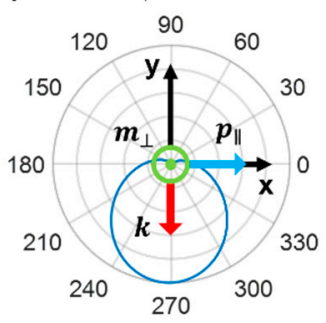

(b)

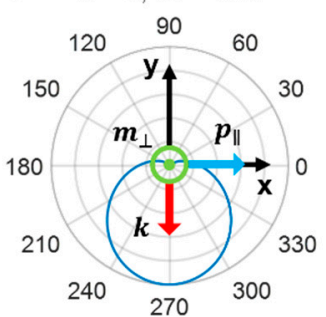

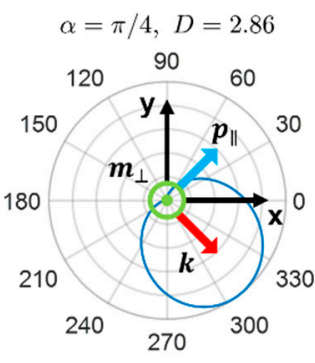

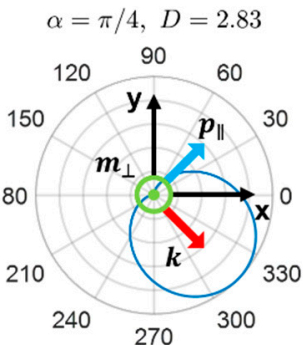

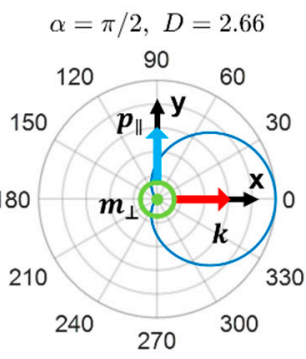

$\alpha=\pi / 2, D=2.85$

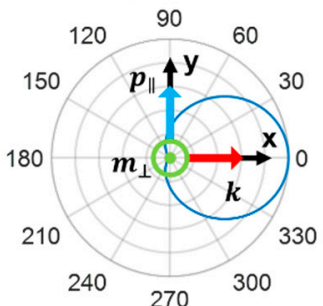

Figure 3. The far-field radiation pattern of the silicon nanodisk under different coherent excitations. The working wavelength is $600 \mathrm{~nm}(\mathbf{a})$ and $633 \mathrm{~nm}(\mathbf{b})$. The polarization angles for $v_{T}(\alpha)$ are (from left to right) $\alpha=0, \pi / 4$, and $\pi / 2$, respectively. Their directivities are calculated to be: $3.02(\alpha=0), 2.86$ $(\alpha=\pi / 4)$, and $2.66(\alpha=\pi / 2)$ for 600 nm excitation, and $2.83(\alpha=0), 2.83(\alpha=\pi / 4)$, and $2.85(\alpha=\pi / 2)$ for $633 \mathrm{~nm}$ excitation, respectively.
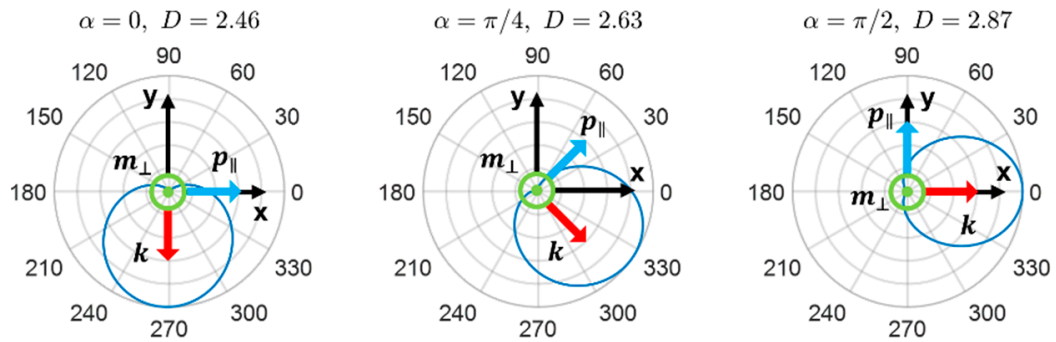

Figure 4. The far-field radiation pattern of the silicon nanodisk immersed in index-matching oil. The working wavelength is $633 \mathrm{~nm}$. The polarization angles for $v_{T}(\alpha)$ are (from left to right) $\alpha=0$, $\pi / 4$, and $\pi / 2$, respectively. The corresponding directivities are: $2.46(\alpha=0), 2.63(\alpha=\pi / 4)$, and 2.87 $(\alpha=\pi / 2)$, respectively.

\section{Conclusions}

In summary, we have proposed an experimentally feasible approach to continuously manipulate the transverse scattering from a single silicon nanodisk, showing numerically that a highly reconfigurable and broadband unidirectional scattering can be obtained by coherent control method. We have demonstrated that the exclusive excitation of resonance mode, commonly unattainable for a single beam, can be achieved by coherent excitations. Our results provide a potential optical manipulation approach in next-generation on-chip integrations and optical communication without the need for movable components.

Supplementary Materials: The following are available online at https://www.mdpi.com/article/10 .3390 / cryst11080920/s1, Figure S1: Schematic of the coherent excitation configuration. Figure S2: Capability test of the coherent control approach.

Author Contributions: Z.C. and H.C. conceived and supervised the study. H.Y., H.Z. and J.L. developed the analytical model and conducted the numerical simulations. The manuscript was written through contributions of all authors. All authors have read and agreed to the published version of the manuscript. 
Funding: This work was financially supported by the National Key Basic Research Program of China (Grant No. 2019YFA0210203), the National Natural Science Foundation of China (Grant Nos. 91963205, 11904420, 61905290), and the Guangdong Basic and Applied Basic Research Foundation (Grant Nos. 2020A1515011329, 2019A1515011355, 202102020579). H.C. acknowledges the support from Changjiang Young Scholar Program.

Data Availability Statement: The data presented in this study are available on request from the corresponding author.

Conflicts of Interest: The authors declare no conflict of interest.

\section{References}

1. Zheng, Z.; Sun, F.; Huang, W.; Jiang, J.; Zhan, R.; Ke, Y.; Chen, H.; Deng, S. Phonon Polaritons in Twisted Double-Layers of Hyperbolic van der Waals Crystals. Nano Lett. 2020, 20, 5301-5308. [CrossRef]

2. Liu, W.; Kivshar, Y.S. Generalized Kerker effects in nanophotonics and meta-optics. Opt. Express 2018, 26, 13085-13105. [CrossRef] [PubMed]

3. Geffrin, J.-M.; Garcia, B.; Gómez-Medina, R.; Albella, P.; Froufe-Pérez, L.S.; Eyraud, C.; Litman, A.; Vaillon, R.; González, F.; Nieto-Vesperinas, M.; et al. Magnetic and electric coherence in forward- and back-scattered electromagnetic waves by a single dielectric subwavelength sphere. Nat. Commun. 2012, 3, 1171. [CrossRef] [PubMed]

4. Fu, Y.H.; Kuznetsov, A.; Miroshnichenko, A.; Yu, Y.F.; Luk'Yanchuk, B. Directional visible light scattering by silicon nanoparticles. Nat. Commun. 2013, 4, 1527. [CrossRef] [PubMed]

5. Gómez-Medina, R.; García-Cámara, B.; Suárez-Lacalle, I.; Gonzalez, F.; Moreno, F.; Nieto-Vesperinas, M.; Sáenz, J.J. Electric and magnetic dipolar response of germanium nanospheres: Interference effects, scattering anisotropy, and optical forces. $J$. Nanophotonics 2011, 5, 053512. [CrossRef]

6. Evlyukhin, A.B.; Reinhardt, C.; Seidel, A.; Luk'Yanchuk, B.S.; Chichkov, B.N. Optical response features of Si-nanoparticle arrays. Phys. Rev. B 2010, 82, 045404. [CrossRef]

7. García-Etxarri, A.; Gómez-Medina, R.; Froufe-Pérez, L.S.; Lopez, C.; Chantada, L.; Scheffold, F.; Aizpurua, J.; Vesperinas, M.N.; Sáenz, J.J. Strong magnetic response of submicron Silicon particles in the infrared. Opt. Express 2011, 19, 4815-4826. [CrossRef]

8. Kuznetsov, A.I.; Miroshnichenko, A.E.; Fu, Y.H.; Zhang, J.; Luk'Yanchuk, B. Magnetic light. Sci. Rep. 2012, 2, 1-6. [CrossRef] [PubMed]

9. Decker, M.; Staude, I.; Falkner, M.; Dominguez, J.; Neshev, D.N.; Brener, I.; Pertsch, T.; Kivshar, Y.S. High-efficiency dielectric Huygens' surfaces. Adv. Opt. Mater. 2015, 3, 813-820. [CrossRef]

10. Bag, A.; Neugebauer, M.; Woźniak, P.; Leuchs, G.; Banzer, P. Transverse Kerker Scattering for Angstrom Localization of Nanoparticles. Phys. Rev. Lett. 2018, 121, 193902. [CrossRef]

11. Shamkhi, H.K.; Baryshnikova, K.V.; Sayanskiy, A.; Kapitanova, P.; Terekhov, P.; Belov, P.; Karabchevsky, A.; Evlyukhin, A.B.; Kivshar, Y.; Shalin, A.S. Transverse Scattering and Generalized Kerker Effects in All-Dielectric Mie-Resonant Metaoptics. Phys. Rev. Lett. 2019, 122, 193905. [CrossRef]

12. Bohren, C.F.; Huffman, D.R. Absorption and Scattering of Light by Small Particles; John Wiley \& Sons: New York, NY, USA, 1983.

13. Zheludev, N.I. Obtaining optical properties on demand. Science 2015, 348, 973-974. [CrossRef]

14. Lee, J.Y.; Huang, L.; Xu, L.; Miroshnichenko, A.E.; Lee, R.-K. Broadband control on scattering events with interferometric coherent waves. New J. Phys. 2021, 23, 063014. [CrossRef]

15. Lee, J.Y.; Chung, Y.-H.; Miroshnichenko, A.E.; Lee, R.-K. Linear control of light scattering with multiple coherent waves excitation. Opt. Lett. 2019, 44, 5310-5313. [CrossRef]

16. Xi, Z.; Wei, L.; Adam, A.J.L.; Urbach, H.P. Broadband active tuning of unidirectional scattering from nanoantenna using combined radially and azimuthally polarized beams. Opt. Lett. 2015, 41,33-36. [CrossRef]

17. Zhang, J.; MacDonald, K.; Zheludev, N. Controlling light-with-light without nonlinearity. Light. Sci. Appl. 2012, 1, e18. [CrossRef]

18. Roger, T.; Vezzoli, S.; Bolduc, E.; Valente, J.; Heitz, J.J.F.; Jeffers, J.; Soci, C.; Leach, J.; Couteau, C.; Zheludev, N.I.; et al. Coherent perfect absorption in deeply subwavelength films in the single-photon regime. Nat. Commun. 2015, 6, 7031. [CrossRef] [PubMed]

19. Shi, J.; Fang, X.; Rogers, E.; Plum, E.; MacDonald, K.; Zheludev, N. Coherent control of Snell's law at metasurfaces. Opt. Express 2014, 22, 21051-21060. [CrossRef] [PubMed]

20. Neugebauer, M.; Woźniak, P.; Bag, A.; Leuchs, G.; Banzer, P. Polarization-controlled directional scattering for nanoscopic position sensing. Nat. Commun. 2016, 7, 11286. [CrossRef]

21. Wei, L.; Zayats, A.V.; Rodríguez-Fortuño, F.J. Interferometric Evanescent Wave Excitation of a Nanoantenna for Ultrasensitive Displacement and Phase Metrology. Phys. Rev. Lett. 2018, 121, 193901. [CrossRef]

22. Chen, J.; Ng, T.F.J.; Lin, Z.; Chan, C.T. Optical pulling force. Nat. Photon. 2011, 5, 531-534. [CrossRef]

23. Pu, M.; Feng, Q.; Wang, M.; Hu, C.; Huang, C.; Ma, X.; Zhao, Z.; Wang, C.; Luo, X. Ultrathin broadband nearly perfect absorber with symmetrical coherent illumination. Opt. Express 2012, 20, 2246-2254. [CrossRef] [PubMed]

24. Karabchevsky, A.; Katiyi, A.; Ang, A.; Hazan, A. On-chip nanophotonics and future challenges. Nanophotonics 2020, 9, 3733-3753. [CrossRef] 
25. Willner, A.E.; Huang, H.; Yan, Y.; Ren, Y.; Ahmed, N.; Xie, G.; Bao, C.; Li, L.; Cao, Y.; Zhao, Z.; et al. Optical communications using orbital angular momentum beams. Adv. Opt. Photon. 2015, 7, 66-106. [CrossRef]

26. Cao, Z.; Ong, H. Momentum-dependent group velocity of surface plasmon polaritons in two-dimensional metallic nanohole array. Opt. Express 2016, 24, 12489-12500. [CrossRef] [PubMed]

27. Kuznetsov, A.; Miroshnichenko, A.; Brongersma, M.L.; Kivshar, Y.S.; Luk'Yanchuk, B. Optically resonant dielectric nanostructures. Science 2016, 354, 2472. [CrossRef]

28. Evlyukhin, A.B.; Reinhardt, C.; Chichkov, B.N. Multipole light scattering by nonspherical nanoparticles in the discrete dipole approximation. Phys. Rev. B 2011, 84, 235429. [CrossRef]

29. Hinamoto, T.; Fujii, M. MENP: An open-source MATLAB implementation of multipole expansion for nanophotonics. OSA Contin. 2021, 4, 1640-1648. [CrossRef]

30. Alaee, R.; Rockstuhl, C.; Fernandez-Corbaton, I. Exact Multipolar Decompositions with Applications in Nanophotonics. Adv. Opt. Mater. 2018, 7, 1800783. [CrossRef]

31. Van De Groep, J.; Polman, A. Designing dielectric resonators on substrates: Combining magnetic and electric resonances. Opt. Express 2013, 21, 26285-26302. [CrossRef]

32. Staude, I.; Miroshnichenko, A.; Decker, M.; Fofang, N.T.; Liu, S.; Gonzales, E.; Dominguez, J.; Luk, T.S.; Neshev, D.N.; Brener, I.; et al. Tailoring Directional Scattering through Magnetic and Electric Resonances in Subwavelength Silicon Nanodisks. ACS Nano 2013, 7, 7824-7832. [CrossRef] [PubMed]

33. Long, S.; McAllister, M.; Shen, L. The resonant cylindrical dielectric cavity antenna. IRE Trans. Antennas Propag. 1983, 31, 406-412. [CrossRef] 